\title{
A CLASS OF ABSTRACT VOLTERRA EQUATIONS, VIA WEAKLY PICARD OPERATORS TECHNIQUE
}

\author{
M. A. Şerban, I. A. Rus And A. Petruşel
}

Abstract. In this paper we consider the following abstract Volterra equations:

$$
x(t)=G(t, g(x)(t), x(t), x(0))+\int_{-t}^{t} K(t, s, x(s), x(h(s))), t \in \mathbb{R}
$$

and

$$
x(t)=G(t, g(x)(t), x(t), x(0))+\int_{-|t|}^{|t|} K(t, s, x(s), x(h(s))) d s, t \in \mathbb{R} .
$$

Using the weakly Picard operator technique we establish existence, data dependence and comparison results for the solution. The derivability of the solutions with respect to a parameter is also studied.

Mathematics subject classification (2010): 47H10, 47N20, 45N05, 45D05, 45M10, 45M99.

Keywords and phrases: L-space, gauge space, weakly Picard operator, abstract Volterra equation, Volterra integral equation, functional-integral equation, solution set, data dependence, comparison theorem, operatorial inequalities.

\section{REFERENCES}

[1] C. Avramescu, Sur l'éxistence des équations integrales dans certaines espaces fonctionnels, Ann. Univ. Scient. Budapesinensis de Roland Eötvös, 13(1970), 19-34.

[2] A. Chiş-Novac, R. PRecup AND I. A. Rus, Data dependence of fixed points for non-self generalized contractions, Fixed Point Theory, 10(2009), 73-87.

[3] C. Corduneanu, Abstract Volterra equation: a survey, Nonlinear Operator Theory, Math. Comput. Modelling, 32 (2000), No. 11-13, 1503-1528.

[4] D. Guo, V. Lakshmikantham and X. Liu, Nonlinear Integral Equations in Abstract Spaces, Kluwer Acad. Publ., Dordrecht, 1996.

[5] D. O'Regan, A. Petruşel, T. P. Petru, Fixed point results for Ciric type contractions on a set with two separating gauge structures, Sci. Math. Jpn., 68(2008), no. 3, 361-369.

[6] A. Petruşel, I. A. Rus, M. A. ŞERBAn, Fixed points for operators on generalized metric spaces, CUBO 10(2008), 45-66.

[7] R. Precup, Methods in Nonlinear Integral Equations, Kluwer Acad. Publ., Dordrecht, 2002.

[8] I. A. RUs, Who authored the first integral equations book in the world, Seminar on Fixed Point Theory, Cluj-Napoca, 1(2000), 81-86.

[9] I. A. RUS, A class of nonlinear functional-integral equations, via weakly Picard operators, Ann. Univ. Craiova, Ser. Mat.-Infor., 28(2001), 10-15.

[10] I. A. Rus, Weakly Picard operators and applications, Seminar on Fixed Point Theory, Cluj-Napoca, 2(2001), 41-58.

[11] I. A. Rus, Picard operators and applications, Scienticae Mathematicae Japonicae, 58(2003), 191219. 
[12] I. A. RUS, The theory of a metrical fixed point theorem: theoretical and applicative relevances, Fixed Point Theory, 9(2008), 541-559.

[13] I. A. Rus, A. Petruşel, G. Petruşel, Fixed Point Theory, Cluj University Press, 2008.

[14] I. A. Rus, A. Petruşel, M. A. ŞERBAn, Fibre Picard operators on gange spaces and applications, Journal for Analysis and its Applications, 27(2008), no. 4, 399-415.

[15] G. SzEP, On an integral equation with deviating argument, Seminar on Fixed Point Theory ClujNapoca, 1(2000), 103-108.

[16] Z. B. TsalyuK, Volterra functional inequalities, Yzv. Vyssh. Ucheb. Zaved, 3(1969), 86-95. 